\title{
Colour modifications and hyperspectral imaging: non-invasive analysis of photo-degraded wood surfaces
}

\author{
Giorgia Agresti, ${ }^{1}$ Giuseppe Bonifazi, ${ }^{2}$ Luca Calienno, ${ }^{3}$ Giuseppe Capobianco, ${ }^{2}$ \\ Angela Lo Monaco, ${ }^{3}$ Claudia Pelosi, ${ }^{1}$ Rodolfo Picchio, ${ }^{3}$ Silvia Serranti ${ }^{2}$ \\ ${ }^{1}$ Department of Cultural Heritage Sciences, University of Tuscia , Largo dell'Università, Viterbo, \\ Italy; ${ }^{2}$ Department of Chemical Engineering Materials \& Environment, Sapienza - Rome \\ University, Rome, Italy; ${ }^{3}$ Department of Agriculture, Forests, Nature and Energy (DAFNE), \\ University of Tuscia, Via San Camillo de Lellis, Viterbo, Italy
}

\begin{abstract}
The aim of this investigation is to study the changes occurring on the surface of poplar wood exposed to solar irradiation, in a controlled environment. Poplar is poor of coloured extractive, so that the surface changes are attributed to the main wood constituents' modifications and the contribution of extraneous substances to holocellulose and lignin can be considered negligible. The poplar wood samples were irradiated in a Solar Box chamber, equipped with a $280 \mathrm{~nm}$ UV filter, until reaching 504 hours. The colour changes were monitored with the reflectance spectrophotometer of X-Rite CA22 according to the CIELAB system. The surface chemical modifications were evaluated by measuring the infrared spectra with a Fourier Transform Infrared spectrometer (FT-IR) operating in DRIFT modality. Hyperspectral imaging (HSI) was also applied to study the surface wood changes by using a SisuCHEMA XL Workstation operating in the SWIR range (1000-2500 $\mathrm{nm})$. The data obtained from the different techniques applied were put in comparison in order to find possible correlations between them also with the aim to evaluate the applicability of the HSI technique to the investigation of wood modifications, in a totally non-invasive modality. The possibility to find a correlation between colour changes and chemical modifications, investigated both with traditional and innovative methodologies, in wood surfaces can have practical application in cultural heritage and contemporary objects.
\end{abstract}

Correspondence: Luca Calienno, Department of Agriculture, Forests, Nature and Energy (DAFNE), University of Tuscia, via San Camillo de Lellis, 01100 Viterbo (Italy). E-mail: luca.calienno@unitus.it

Key words: Poplar; Colour measurement, FT-IR spectroscopy, Hyperspectral imaging; wood.

(C) Copyright G. Giorgia et al., 2013

Licensee PAGEPress, Italy

Journal of Agricultural Engineering 2013; XLIV(s2):e5

doi:10.4081/jae.2013.s2.e5

This article is distributed under the terms of the Creative Commons Attribution Noncommercial License (by-nc 3.0) which permits any noncommercial use, distribution, and reproduction in any medium, provided the original author(s) and source are credited.

\section{Introduction}

The focus of this work is to study the surface modifications of poplar (Populus spp.) wood by reflectance spectrophotometry, Fourier Transform Infrared (FT-IR) spectroscopy and HyperSpectral Imaging (HSI) in order to understand the mechanisms that cause surface changes due to photo-degradation and to suggest possible solutions to avoid the degradation phenomena. The choice of poplar was due to its widespread use in Italy for the creation of statues, ceilings, furniture, doors, painted panels, etc.

In cultural heritage the monitoring of wood surfaces during light exposure should be performed through non-destructive methods to avoid the paradox of damaging a work of art while monitoring its preservation state (Marengo, et al. 2003; Marengo, et al. 2004). For this reason colour measurements were chosen as possible method to evaluate wood surface changes.

Since colour changes on wood surfaces are due to photo degradation of its chemical constituents, the study of the relationship between CIELAB colour changes and changes in chemical composition due to irradiation is of practical importance both in cultural heritage and in contemporary artefacts and objects (Pandey, 2005)

This work starts from previous studies, developed by the same authors, regarding colour measurements on poplar and chestnut wood (Lo Monaco, et al. 2011a; Lo Monaco, et al. 2011b; Genco, et al. 2011; Pelosi et al. 2013). The novelty of this work, in respect of the previous ones, is the attempt to verify the possible correlations existing between colorimetric and chemical data in the perspective to develop simple, fast and reliable techniques to detect wood surface modifications.

The first studies dealing with the application of multi-spectral and HSI based techniques, in the visible region, to wood date back to 1941 (Pillow, 1941) where this approach, based on classical spectra collection, was utilised to identify compression wood in conifers. Compression wood appears more opaque, in comparison with normal wood that appears translucent. Methods based on NIR imaging were thus developed and applied to classify wood (i.e. compression and opaque) (Nystrom and Hagman, 1999) and further "refined" to perform regions mapping (i.e. bark, compression and normal wood) (Noordam, et al. 2005). In the recent years the utilisation of HSI based techniques increased considerably, also in the field of wood studies where they are used especially to perform the chemical surface mapping for obtaining the distribution of lignin, galactose and glucose in different wood species (Thumm, et al. 2010).

In this work, the classical colorimetric investigation procedures, the chemical analysis and the proposed HSI based techniques are compared in order to verify the possibility to use colour measurements as a quick and simple method to control the surface modifications, map- 
ping (i.e. pixel by pixel) the wood sample surface.

The fulfilment of this goal was achieved by examining the correlations between classical colorimetric and chemical data with wood surface spectral characteristics detected in the i) VIS-NIR (400-1000 nm) and ii) SWIR (1000-2500 $\mathrm{nm}$ ) wavelength ranges. This approach is of great interest in the perspective to set up efficient, robust, non invasive and not destructive analytical tools addressed to evaluate status and modifications of photo-degraded wood surfaces.

\section{Materials and Methods}

\section{Colour monitoring and FT-IR analysis}

Wood samples were obtained by a single board of poplar. After cutting, the samples were stored in darkness in a conditioned room at $65 \%$ relative humidity and a temperature of $22^{\circ} \mathrm{C}$ to reach the $12 \%$ of moisture content.

The samples dimensions for the colour examination were $235 \mathrm{x} 50 \mathrm{x}$ $50 \mathrm{~mm}$.

To perform the FT-IR analysis directly on wood surface, circular slices, diameter $10 \mathrm{~mm}$ and $2 \mathrm{~mm}$ thick, were obtained from the specimens of poplar. The dimensions of the slices were suitable for the FTIR diffuse reflectance accessory. The same slices were used to perform the HSI measurements.

The accelerated ageing of the samples was performed in a Model 1500E Solar Box (Erichsen Instruments $\mathrm{GmbH} \& \mathrm{Co}$ ), in order to simulate the sunlight exposition. The samples were exposed in the Solar Box chamber from 1 to $504 \mathrm{~h}$ at $550 \mathrm{~W} / \mathrm{m}^{2}, 55^{\circ} \mathrm{C}$ and the UV filter at 280 $\mathrm{nm}$.

After exposure for a given length of time the samples were removed from the Solar Box chamber and the colour was measured using an XRite CA22 reflectance spectrophotometer. The characteristics of the colour measuring instrument are the following: colour scale CIEL*a*b*; illuminant D65; standard observer $10^{\circ}$; geometry of measurement $45^{\circ} / 0^{\circ}$; spectral range $400-700 \mathrm{~nm}$; spectral resolution $10 \mathrm{~nm}$; measurement diameter $4 \mathrm{~mm}$; white reference supplied with the instrument. The CIELAB colour system was used where $\mathrm{L}^{*}$ describes the lightness while $\mathrm{a}^{*}$ and $\mathrm{b}^{*}$ describe the chromatic coordinates on the green-red and blue-yellow axes, respectively. Measurements were taken at the following hour intervals: $0,1,2,3,4,5,6,7,8,9,10,11,12$, $24,48,72,96,120,144,168,216,312,408$ and $504 \mathrm{~h}$. Ninety colour measuring points were chosen and three measures for each point were performed, according to Normal 14/83, so that two hundred and seventy measurements were realized at each exposure time.

Infrared spectra were obtained using a Nicolet Avatar 360 Fourier transform spectrometer. For each sample 128 scans were recorded in the 4000 to $400 \mathrm{~cm}^{-1}(2500-25000 \mathrm{~nm})$ spectral range in diffuse reflection modality (DRIFT) with a resolution of $4 \mathrm{~cm}^{-1}$. Spectral data were collected with OMNIC 8.0 (Thermo Electron Corporation) software.

To obtain the FT-IR spectra, sample slices were directly inserted in the FT-IR diffuse reflectance accessory unit. As background the spectrum of the $\mathrm{KBr}$ powder was used. The spectra were collected in DRIFT modality directly on the wood slices in order to avoid analysing also the unaltered wood. In fact, wood is a good light absorber for infrared, visible and ultraviolet light (Kataoka and Kigushi 2001; George, et al. 2005). In particular the UV component of light is totally absorbed in a $75 \mu \mathrm{m}$-thick layer (Hon and Shiraishi 2001). Since the change caused by irradiation appears in a thin surface layer, it is not easy to remove the proper thin layer for pellet preparation, as suggested also by other authors (Tolvaj, et al. 2011).

FT-IR spectra were recorded at the following time intervals: $0,6,12$,
24, 48, 72, 96, 120, 144, 168, 216, 312, 408 and 504 hours.

Band assignment was made according to literature references (Moore and Owen 2001; Chang, et al. 2002; Colom, et al. 2003; Pandey and Pitman 2003).

Peak heights were measured using OMNIC software according to the method described in the literature (Pandey and Pitman 2003).

The colour and FTIR data were analyzed with the StatSoft (® Statistica 2010 advanced statistics software. As a first step, data distribution was plotted and visually checked for normality. Differences between treatments were checked with the standard paired t-test, with ANOVA and M-ANOVA analysis. Post-hoc tests were conducted with Tukey HSD test method. Linear and non-linear regression analysis was used to develop prediction models.

In particular, two-way ANOVA was applied to the infrared peak values to test if significant differences in relation to the samples and to the time could be found.

\section{Hyperspectral analyses}

Hyperspectral analyses were carried out in 2 steps: a $1^{\text {st }}$ step addressed to analyse degraded wood surfaces in the wavelength interval 400-1000 nm (VIS-NIR) and a $2^{\text {nd }}$ in which analyses were carried out in the interval 1000-2500 nm (SWIR). Investigations have been developed with 2 different sensing devices, both from SPECIM Ltd, Finland: i) an ImSpector ${ }^{\mathrm{TM}}$ V10E acting in the range $400-1000 \mathrm{~nm}$, with a spectral sampling/pixel of $2.8 \mathrm{~nm}$, a dispersion of $97.5 \mathrm{~nm} / \mathrm{mm}$ and F/2.4 numerical aperture, coupled with a CCD camera (780x580 pixels) and ii) a Specim SISUChema XL ${ }^{\mathrm{TM}}$, embedding an ImSpector ${ }^{\mathrm{TM}} \mathrm{N} 25 \mathrm{E}$ acting in the range $1000-2500 \mathrm{~nm}$, with a spectral sampling/pixel of 6.3 $\mathrm{nm}$, coupled with a MCT camera (320x240 pixels). Pixel resolution was 12 bits for the first device and 14 bits for the second, respectively.

For the $1^{\text {st }}$ set of acquisitions $(400-1000 \mathrm{~nm})$ the $\mathrm{ImSpector}{ }^{\mathrm{TM}} \mathrm{V} 10 \mathrm{E}$ was installed on a Leica M205C stereomicroscope. The energysing source was constituted by MI-150 Dolan Jenner fiber optic device equipped with a dicroic lamp. For the $2^{\text {nd }}$ set of acquisitions (1000-2500 $\mathrm{nm}$ ) the SISUChema XL ${ }^{\mathrm{TM}}$ was used, equipped with macro lens allowing the acquisition of wood samples with a resolution of $30 \mathrm{~m} / \mathrm{pixel}$. Images were acquired scanning each investigated sample line by line.

The calibration of the procedures was performed recording two black and white reference images. Certified standards were used. Black image $(B)$ was acquired to remove the camera sensor dark current effect. White reference image $(W)$ was acquired using a white ceramic tile, calibrated with a NPL Spectralone ${ }^{\circledR}$ specimen, in the same condition for the raw image acquisition. The image correction was thus performed adopting the following equation:

$$
I=\frac{I_{0}-B}{W-B} \times 100
$$

where $I$ is the corrected hyper-spectral image in a unit of relative reflectance (\%), $I_{0}$ is the original hyperspectral image, $B$ is the black reference image ( $\sim 0 \%$ reflectance) and $W$ is the white reference image ( $\sim 99.9 \%$ reflectance). All the corrected images were then used to perform the HSI based analysis, that is to extract spectral information and to select the effective wavelengths for the final classification purposes.

Spectral data analysis was carried out adopting standard chemometric methods (Geladi, et al. 2007; Otto 1999), by the PLS_Toolbox (Version 6.5.1, Eigenvector Research, Inc.) running inside Matlab ${ }^{\circledR}$ (Version 7.11.1, The Mathworks, Inc.). Raw spectra were preliminary cut, at the beginning and at the end of the investigated wavelength range, to eliminate unwanted effects due to lighting/background noise. Wavelength reduction, in VIS-NIR, was from 121 to $81 \mathrm{~nm}$ so that the investigations was carried out in the spectral range $500-900 \mathrm{~nm}$. As 
Table 1. Nonlinear regression analysis applied to the chromatic coordinates at different times of exposure in Solar Box for poplar samples.

\begin{tabular}{lccccccccc} 
Value & Intercept & Significance & Time & Significance & time2 & Significance & R2adj. & Significance \\
$\mathrm{L}^{*}$ & 83.002 & $* * *$ & -0.045 & $* * *$ & 0.00004 & $* * *$ & 0.816 & $* * *$ \\
$\mathrm{a}^{*}$ & 1.569 & $* * *$ & 0.032 & $* * *$ & -0.00003 & $* *$ & 0.954 & $* * *$ \\
\hline $\mathrm{b}^{*}$ & 20.108 & $* * *$ & 0.00679 & $* * *$ & -0.00008 & $* *$ & 0.857 & $* *$ \\
\hline
\end{tabular}

regards SWIR, the reduction was from 256 to $240 \mathrm{~nm}$ resulting in a spectral range 1005-2500 nm. Different pre-treatments, e.g.: De-trend 1st polynomial, Mean-center, $1^{\text {st }}$ Derivative, Standard Normal Variation, Generalized Least Square Weighting (GLSW) and Baseline techniques, were applied to classify the different investigated samples.

Principal Component Analysis (PCA) was used to decompose the "processed" spectral data into several Principal Components (PCs) (linear combinations of the original spectral data) embedding the spectral variations of each collected spectral data set. A reduced set of factors is produced. Such a set can be used for discrimination, since it provides an accurate description of the entire dataset.

Partial Least Squares Discriminant Analysis (PLS-DA) was used to find a model able to perform an optimal discrimination among classes of samples and to predict new images. PLS-DA is a supervised classification technique, requiring a prior knowledge of the data (Barker and Rayens 2003). The result of PLS-DA applied to hyper-spectral images is a "prediction map", where the class of each pixel can be identified using color mapping.

\section{Results and discussion}

\section{Colour monitoring and FT-IR analysis}

Colour variability of poplar wood was widely discussed elsewhere suggesting the necessity to perform a high number of measurements in order to avoid this variability (Lo Monaco, et al. 2011; Ferreira, et al. 2012). M-ANOVA and Tukey tests were applied to the chromatic coordinates as function of irradiation times, until 504 hours of exposure. These tests underline that $\mathrm{L}^{*}, \mathrm{a}^{*}$ and $\mathrm{b}^{*}$ undergo statistically significant changes after the exposure times, apart from the first hours of irradiation suggesting the possibility to start the measurements directly after 6 hours of irradiation

In particular $\mathrm{L}^{*}$ value decreases from 84.3 to 71.6 . The $\mathrm{a}^{*}$ and $\mathrm{b}^{*}$ coordinates increase from 2.0 to 9.5 and from 16.8 to 33.3 respectively. In general it is possible to state that the greatest change occurs within the first $24 \mathrm{~h}$ of exposure and around the $460 \mathrm{~nm}$ wavelength ( Reflectance\% $=-28.3$ ) which corresponds in violet/blue shades.

A nonlinear regression analysis was applied to the chromatic coordinates at different time of exposure in Solar Box in order to evaluate the statistical significance of the experimental data. The results reported in Table 1 demonstrate that the various components exhibit a high statistical significance concerning every polynomial function used for the analysis.

A nonlinear regression analysis with three dependent variables was applied to the time in function of colour coordinates. The results shown in Table 2 underline the highly statistical significance of the obtained measures regarding the dependent variable time (T) as function of the colour coordinates $\left(\mathrm{L}^{*}, \mathrm{a}^{*}, \mathrm{~b}^{*}\right)$.

The irradiating-time dependent diffuse reflectance FT-IR spectra are shown in Figure 1. It can be observed that the intensity of the bands at $1507 \mathrm{~cm}^{-1}, 1595 \mathrm{~cm}^{-1}$ and $1464 \mathrm{~cm}^{-1}$, associated to lignin, decrease dur-
Table 2. Non linear regression analysis applied to the time as function of the chromatic coordinates.

\begin{tabular}{lcc} 
Poplar samples & \multicolumn{1}{c}{ B } & Significance \\
Intercept & 1732.326 & $* * *$ \\
$\mathrm{~L}^{*}$ & -56.232 & $* * *$ \\
\hline $\mathrm{a}^{*}$ & 16.154 & $* * *$ \\
$\mathrm{~b}^{*}$ & 24.495 & $* * *$ \\
$\mathrm{~L}^{* 2}$ & 0,381 & $* * *$ \\
$\mathrm{a}^{* 2}$ & 4.376 & $* * *$ \\
\hline $\mathrm{b}^{* 2}$ & -0.520 & $* * *$ \\
$\mathrm{R}^{2} \mathrm{adj} .=0.947 ; \mathrm{p}<0.001$ & \\
$\mathrm{~T}=1732.3-56.2 \mathrm{~L}^{*}+16.2 \mathrm{a}^{*}+24.5 \mathrm{~b}^{*}+0.4 \mathrm{~L}^{*} 2+4.4 \mathrm{a}^{*} 2$ & \\
\hline
\end{tabular}

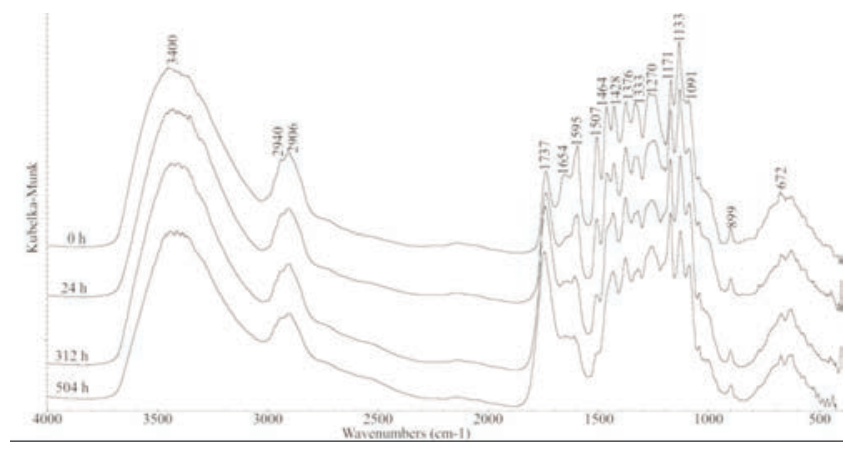

Figure 1. Diffuse reflectance spectra of poplar slices measured at chosen exposure times.
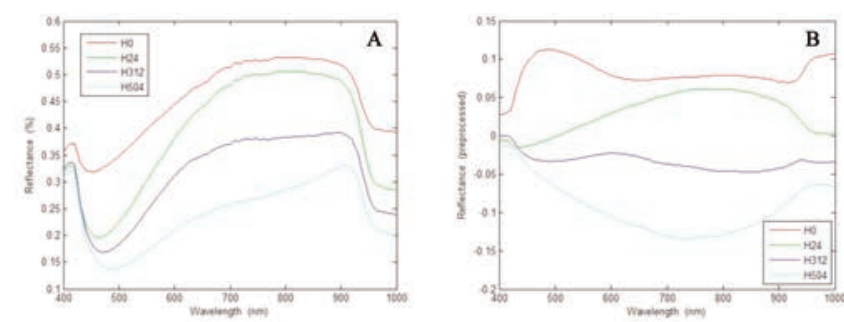

Figure 2. Acquired (2A) and pre-processed (2B) spectra of the different analysed wood slices, in the VIS-NIR field. Spectra pre-processing was carried out adopting a GLSW procedure. 
Table 3. Two-way ANOVA test applied to the peak ratios in relation to the poplar samples and to the irradiation time.

\begin{tabular}{lccccc}
\hline Peak ratio & Measures Nr. & Average & Std. Dev. & Samples differences & Time differences \\
$\mathrm{I}_{1507} / I_{1376}$ & 42 & 0.827 & 0.490 & No significance & $* *$ \\
$\mathrm{I}_{1740} \mathrm{I}_{1376}$ & 42 & 3.210 & 1.030 & No significance & $*$ \\
\hline $\mathrm{I}_{1507} / I_{1737}$ & 42 & 0.326 & 0.302 & No significance & $* *$ \\
\hline
\end{tabular}

ing photo-degradation. This is accompanied by an increase in the intensity of the band at $1737 \mathrm{~cm}^{-1}$, due to carbonyl absorption. The intensities of the peak associated to carbohydrates at $1376 \mathrm{~cm}^{-1}$ is not significantly affected by irradiation, so this band has been used as internal reference to evaluate the lignin decay.

In order to determine the rate of lignin decay and carbonyl formation, the intensities of the lignin band at $1507 \mathrm{~cm}^{-1}$, the carbonyl band at $1737 \mathrm{~cm}^{-1}$ and the carbohydrate reference band at $1376 \mathrm{~cm}^{-1}$ were calculated (Pandey and Pitman 2003). Then the relative change in ratio of lignin/carbohydrate bands at different exposure times were derived and statistically evaluated.

In particular, two-way ANOVA applied to the infrared peak (Table 3) shows that the differences in the peak ratio with respect to the exposure times are highly significant concerning the $I_{1507} / I_{1376}$ and $I_{1507} / I_{1737}$ ratios. The difference is only significant as regards the $\mathrm{I}_{1737} / \mathrm{I}_{1376}$ ratio.

Nonlinear regression analysis was also applied to the peak ratio at the different times of exposure in Solar Box. The results are shown in Table 4.

The lignin/carbohydrate intensity ratio decreased with the exposure times and after $48 \mathrm{~h}$ of irradiation the experimental value of the $\mathrm{I}_{1507} / \mathrm{I}_{1376}$ ratio decreased to about $50 \%$ of its original value.

The relative increase of the carbonyl groups during photo-irradiation was calculated by considering the ratio of intensity of carbonyl band at $1737 \mathrm{~cm}^{-1}$ against reference peak at $1376 \mathrm{~cm}^{-1}$. The intensity of the band of the carbonyl groups increases as function of irradiation time. This can be explained with the liberation of $\mathrm{C}=0$ groups of degraded lignin. As the carbonyl groups increase during the photo-ageing whereas the lignin band decreases, the ratio of intensity of the bands at 1507 and $1737 \mathrm{~cm}^{-1}$ undergoes a fast and significant decrease after few hours of exposure. In fact, after 12 hours of exposure the experimental value of the $I_{1507} / I_{1737}$ ratio decreased to about $50 \%$ of its original value.

The regression analysis clearly demonstrates that for the $I_{1507} / I_{1376}$ and $I_{1507} / I_{1737}$ peak ratios a statistically significant regression has been derived from the experimental data. In the case of the $I_{1737} / I_{1376}$ peak ratio the regression has a low statistical significance.

At last the colour changes of wood during irradiation were correlated with lignin decay and the formation of carbonyl groups produced by the photo-degradation process. In fact, as the colour changes of the surfaces are mainly due to the liberation of $\mathrm{C}=0$ groups due to photodegradation of lignin, there would be a correlation between the colour coordinates and the relative intensity of the infrared peaks associated to lignin. The results showed in Table 5 demonstrate that the lignin decay is related to photo-induced colour changes of wood surfaces.

\section{Hyperspectral imaging on the wood slices}

Investigations in the VIS-NIR wavelength range. The acquired and the pre-processed spectra for the different irradiated wood slices, at time 0 (H0), 24 (H24), 312 (H312) and 504 hours (H504) of exposure are shown in Figure 2. The Figure 3 shows the false colour images, as resulting from hypercube data structure. The results of PCA are shown in Figure 4. The spectral data of the four wood samples are clustered into four distinct groups according to their spectral signatures.

A discrimination between the wood samples characterized by differ-
A

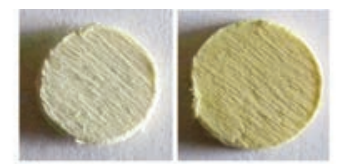

B

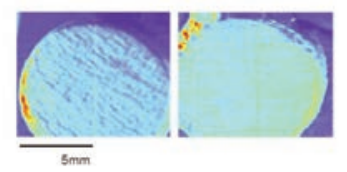

HO
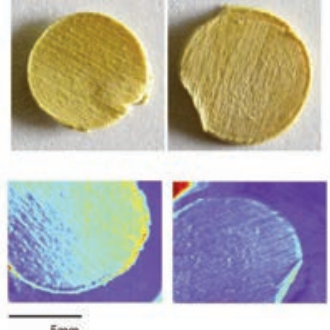

H312

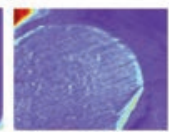

H504

Figure 3. Images of the investigated poplar samples used to build the model. A: acquired images and B: images resulting from hypercube data rendering (i.e. false colours) according to samples spectral response in the VIS-NIR range.

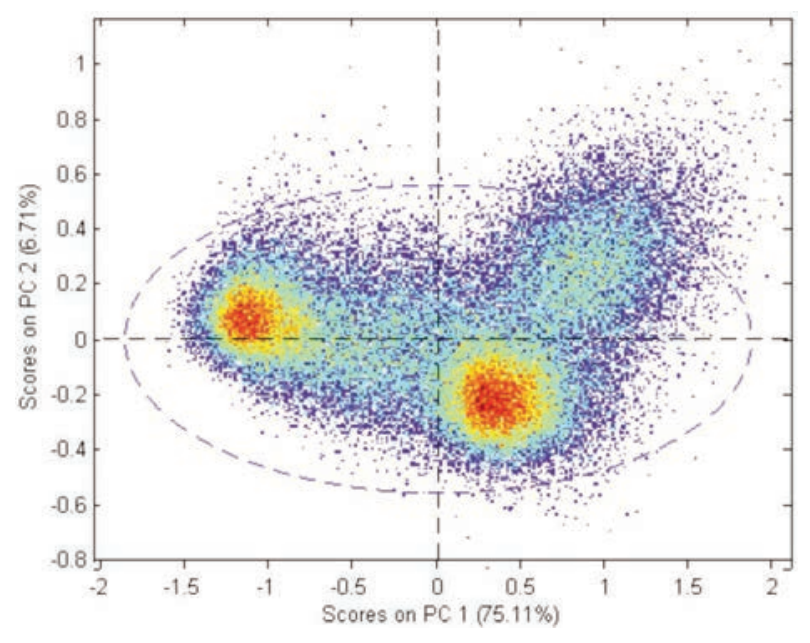

Figure 4. Score plot (PC1 vs PC2), obtained using 81 wavelengths, based on average HSI spectral signatures in the VIS-NIR wavelength region $(400-900 \mathrm{~nm})$ for the analysed poplar wood samples.

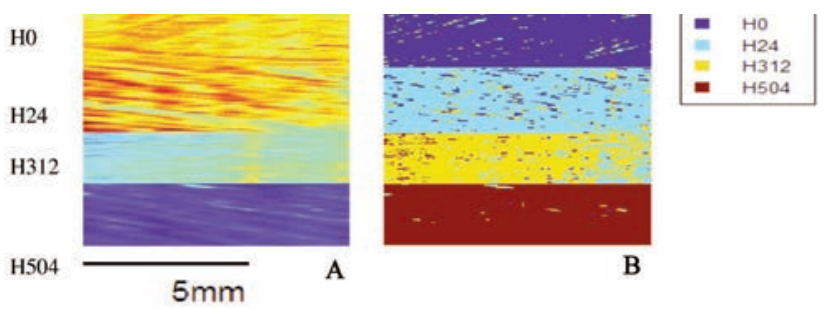

Figure 5. Reconstructed sample image, obtained combining the four different selected ROIs inside the different wood samples, and utilised to perform the hyperspectral based PLS-DA model validation (A). PLS-DA prediction (most probable class prediction) (B). 
Table 4. Nonlinear regression analysis for the peak ratio at the different times of exposure in Solar Box of poplar samples.

\begin{tabular}{|c|c|c|c|c|c|c|c|}
\hline Value & Coefficient & Description & Significance & Time coefficient & Significance & $\mathbf{R}^{2}$ adj. & Significance \\
\hline $\mathrm{I}_{1507} / \mathrm{I}_{1376}$ & 1.383 & e coefficient & $* * *$ & -0.005 & $* * *$ & 0.865 & $* * *$ \\
\hline Regression & \multicolumn{7}{|c|}{$\mathrm{I}_{1507 /} / \mathrm{I}_{376}=1.383 \mathrm{e}^{-0.005 \mathrm{t}}$} \\
\hline $\mathrm{I}_{1737} / \mathrm{I}_{1376}$ & 2.808 & Intercept & $* * *$ & 0.003 & $*$ & 0.129 & $*$ \\
\hline Regression & \multicolumn{7}{|c|}{$\mathrm{I}_{1737} / \mathrm{I}_{1376}=2.808+0.003 \mathrm{t}$} \\
\hline $\mathrm{I}_{1507} / \mathrm{I}_{1737}$ & $\begin{array}{l}0.995 \\
-0.109\end{array}$ & $\begin{array}{c}\text { Intercept } \\
\text { Square root coefficient }\end{array}$ & $\begin{array}{l}* * * \\
* * *\end{array}$ & 0.0031 & $* * *$ & 0.924 & $* * *$ \\
\hline & & & Regression & $\mathrm{I}_{1507} / \mathrm{I}_{733 \mathrm{t}}=0.995+0.0031 \mathrm{t}-0.109 \mathrm{t}^{0.5}$ & & & \\
\hline
\end{tabular}

Table 5. Nonlinear regression analysis applied to the peak ratio as function of the chromatic coordinates $\mathbf{L}^{*} \mathbf{a}^{*} \mathbf{b}^{*}$ in poplar wood.

\begin{tabular}{|c|c|c|c|c|c|}
\hline & & & & $\mathrm{I}_{150 /} / \mathrm{I}_{1737}$ & \\
\hline & B & Significance & B & & Significance \\
\hline Intercept & 1.4582 & $* * *$ & Intercept & -12.420 & $* * *$ \\
\hline $\mathrm{L}^{2 *}$ & 0.000092 & $* * *$ & $\mathrm{~L}^{2 *}$ & 0.0017 & $* * *$ \\
\hline$a^{2 *}$ & -0.00373 & $* * *$ & $a^{2 *}$ & -0.0072 & $* * *$ \\
\hline $\mathrm{b}^{2 *}$ & -0.001328 & $* * *$ & $b^{2 *}$ & 0.0032 & $* * *$ \\
\hline
\end{tabular}

$\mathrm{R}^{2}$ adj. $=0.999 \quad \mathrm{R}^{2} \mathrm{adj} .=0.995$

$\mathrm{I}_{1507} / \mathrm{I}_{376}=1.4582+0.000092 \mathrm{~L} * 2-0.00373 \mathrm{a}^{* 2}-0.001328 \mathrm{~b}^{* 2} 11507 / 11737=-12.420+0.0017 \mathrm{~L}^{* 2}-0.0072 \mathrm{a}^{*} 2+0.0032 \mathrm{~b}^{* 2}$

ent exposure times is obtained, especially concerning $\mathrm{H} 0, \mathrm{H} 24$ and H504, whereas H312 is more dispersed between H24 and H504.

The PLS-DA based classification was calculated using the same pretreatment technique adopted to perform PCA (GLSW) analyses for reference class definition. Starting from 4 new samples, the same approach previously outlined was thus applied, that is: i) construction of a sample image to verify PLS-DA model, starting from four different ROIs selected inside the wood samples in order to avoid "border effects"; ii) selection of ROIs, inside the reconstructed sample image, to collect spectra representative of the different irradiated wood slices, to validate the model.

The results, as prediction images, indicate that the PLS-DA models allow obtaining a good classification of all the samples (Figure 5). Such results can be evaluated taking into account the values of sensitivity and specificity parameters obtained. These parameters range from 0 to1, being 1 the ideal value for a prediction model. Sensitivity is defined as the proportion of class members correctly classified. Specificity refers to the proportion of non-class members correctly classified.

The sensitivity and specificity of the PLS-DA model are calculated both for Calibration and Cross-Validation. The values of sensitivity and specificity range between 0.75 (H312) and 0.996 (H0) for Calibration and between 0.74 (H312) and 0.996 (H0) for Cross-Validation. Good values of sensitivity and specificity are obtained for the classification of the different time exposed wood samples, indicating the robustness of the model. The sample exposed 312 hours was confirmed as the worst classified according to the indications obtained during the explorative analysis based on PCA previously carried out and described.

\section{Hyperspectral imaging on the wood slices}

Investigations in the SWIR wavelength range. The acquired and the pre-processed spectra for the different irradiated wood slices, at time 0 (H0), 24 (H24), 312 (H312) and 504 hours (H504) of exposure are shown in Figure 6. In the SWIR range both cellulose and lignin are characterised by absorption bands corresponding to specific wavelengths, that is $1220,1480,1930,2100,2280,2340$ and $2480 \mathrm{~nm}$ for cellulose and 1450, 1680, 1930, 2270, 2330, 2380 and $2500 \mathrm{~nm}$ for lignin (Van der Meer and Jong 2002).
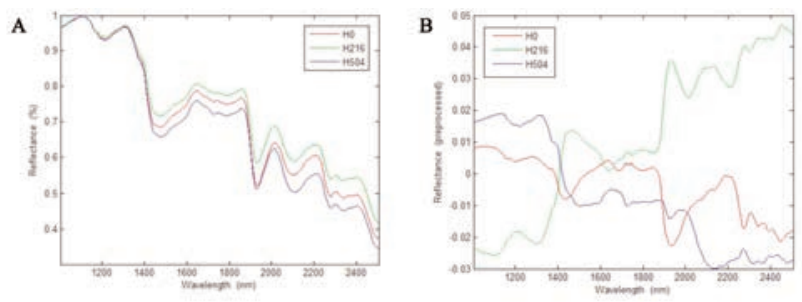

Figure 6. Acquired (A) and pre-processed (B) spectra of the different analysed wood slices, in the SWIR field. Spectra pre-processing was carried out adopting a GLSW procedure.

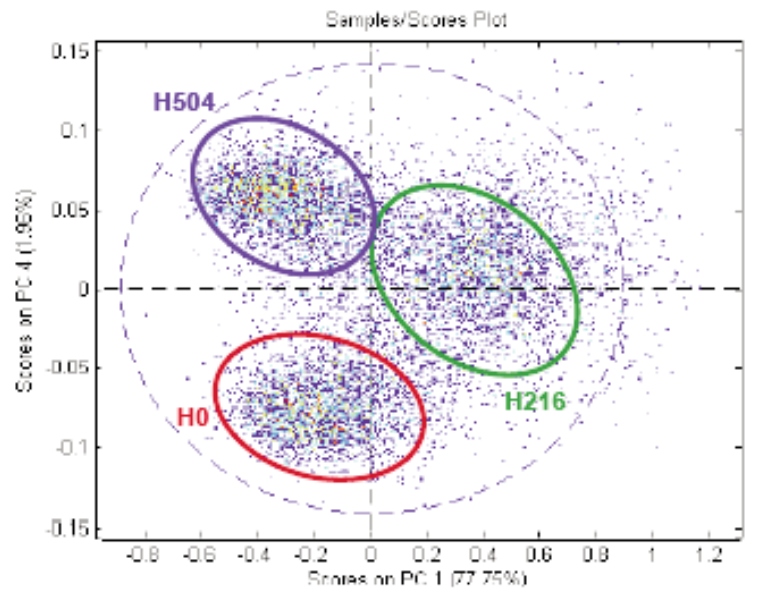

Figure 7. Score plot (PC1 vs PC2), obtained using 240 wavelengths, based on average HSI spectral signatures in the SWIR wavelength region (1005$2500 \mathrm{~nm}$ ), of the analysed poplar wood samples. 
A

Ho

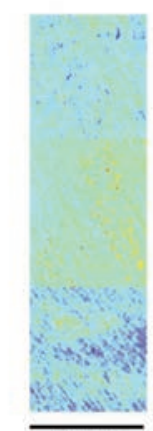

$5 \mathrm{~mm}$
B

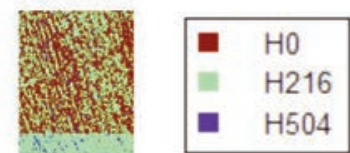

H216

H504

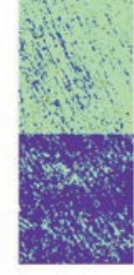

\section{4}

Figure 8. Reconstructed sample image, obtained combining three different ROIs inside the different wood samples, and utilised to perform the hyperspectral based PLS-DA model validation (A). PLS-DA prediction (most probable class prediction) (B).

The strong absorptions at around $1460 \mathrm{~nm}$ and $1930 \mathrm{~nm}$ can be attributed to the combination modalities of $\mathrm{OH}$ in water molecules. It should be noted that the water chemical bond vibration around 1460 $\mathrm{nm}$ may overlap with those of other $\mathrm{OH}$ groups in cellulose molecules or of $\mathrm{CH}_{2}$ groups in lignin molecules (Van der Meer and Jong 2002), making difficult the accurate band assignment in this region. The weak spectral feature around $1730 \mathrm{~nm}$ may be attributed to the presence of $\mathrm{OH}$ bond of water molecule in cellulose/lignin and the weak absorption at $1790 \mathrm{~nm}$ to $0-\mathrm{H}$ stretching in water molecules. The mean absorptions in the 2000-2500 $\mathrm{nm}$ wavelength interval can be attributed to the various configurations of the $\mathrm{C}-\mathrm{H}, \mathrm{C}=\mathrm{O}, \mathrm{C}=\mathrm{C}$, and $-\mathrm{COOH}$ functional groups in cellulose and lignin.

Spectra have been acquired performing a preliminary background removal as, differently from the VIS-NIR case; spectral data collection and analysis have been carried out with reference to the entire surface of each wood sample.

The results of PCA, applied to the average spectral signature of the investigated wood samples are shown in Figure 7. The greater part of the variance was captured by the first two PCs. PC1 and PC2 explained $77.75 \%$ and $1.96 \%$ of the variance, respectively. Spectral data of the 3 wood samples are clustered into 3 distinct groups according to their spectral signatures. A discrimination between the wood samples characterized by different exposure times is thus obtained.

The PLS-DA model was developed, as in the VIS-NIR study, to perform a prediction (i.e. recognition/classification) on unknown wood samples characterised by different exposure times. The PLS-DA based classification was calculated using the same pre-treatment technique adopted to perform PCA (GLSW) analysis for reference class definition. Starting from 3 new samples the following approach was applied: i) construction of a sample image to verify PLS-DA model, performing background elimination, ii) collection of all the spectra related to each sample in order to validate the model. The results indicate that the PLSDA models allowed to reach, as in the VIS-NIR range, a good recognition/classification, according to their irradiation times for the 3 investigated poplar samples (Figure 8).

The sensitivity and specificity of the PLS-DA model, based on all 240 wavelengths, are calculated both for Calibration and Cross-Validation. The values of sensitivity and specificity range between 0.896 (H24) and 0.993 (H0) for Calibration and between 0.868 (H24) and 0.993 (H0).

\section{Conclusions}

The study of colour changes in poplar samples showed the wood surface colour undergoes an important variation due to photo-irradiation. The greatest changes occur within the first 24 hours and they are mainly due to $\mathrm{L}^{*}$ decrease and $\mathrm{b}^{*}$ increase. M-ANOVA and Tukey tests underline that $\mathrm{L}^{*}, \mathrm{a}^{*}$ and $\mathrm{b}^{*}$ undergo statistically significant changes after the exposure times further demonstrating that surface wood colour remarkably changes due to ligth exposure. The regression analysis, applied both to the chromatic coordinates as function of the time and vice versa, underlines the highly statistical significance of the experimental data.

FT-IR spectroscopy allowed to investigate the rate of photo-degradation of wood surface due to lignin oxidation. The rate of photo-degradation of wood surface was investigated by studying the lignin/carbohydrate intensity ratio as function of time. The results put in evidence that lignin degrades quickly: after 48 hours of irradiation the lignin/carbohydrate ratio $\left(\mathrm{I}_{1507} / \mathrm{I}_{1376}\right)$ decreased to about $50 \%$ of its original value. The regression analysis applied to the infrared peak ratios demonstrated that the differences between the values at the various irradiation times are statistically significant, apart from the $I_{1737} / I_{1376}$ ratio. Moreover a correlation between the colour changes and the chemical modifications was investigated in order to assess the applicability of colorimetric measurements in totally non-invasive modality on the works of art.

The most important result was that a correlation of the colour changes may be derived with the photo-degradation of lignin obtained by FTIR analysis. This finding demonstrates that non-invasive colour measurements can be used to evaluate the photo-degradation of wood. In particular the regression analysis showed that the lignin decay is related to the photo-induced colour variations of wood surfaces expressed by the $\mathrm{L}^{*} \mathrm{a}^{*} \mathrm{~b}^{*}$ coordinates.

Concerning hyperspectral imaging, the detected spectral features showed in the VIS-NIR range are mainly linked to the spectral shape (i.e. colour variation), whereas in the SWIR range cellulose and lignin affect both shape and reflectance levels.

The PCA applied to hyperspectral images acquired in the wavelength range 400-1000 $\mathrm{nm}$ highlighted a significant variation between samples not exposed and exposed after 24 hours. The variations between samples irradiated for 312 and 504 hours are lower, if compared with the previous ones, in any case the results showed that the colour changes is correlated with the lignin photo-degradation. Concernign the SWIR, the PCA demonstrated a correlation between sample spectral profile characteristics and the exposure times.

The application of partial least squares regression (PLS-DA) allowed to perform an good classification and recognition of different irradiated samples in both the investigated wavelength ranges. The proposed approach clearly showed as a correlation can be established between color variation and wood degradation in the VIS-NIR; furthermore in the SWIR range wood surface chemical changes can be assessed.

The results obtained show that colour is a promising tool for non invasive assessment of the chimical variation of wood surface in order to monitor both historycal wood artefacts and contemporary wood objects.

\section{References}

Barker M., Rayens W.2003.Partial least squares for discrimination, J. Chemom. 17: 166-173.

Chang H.T., Yeh T.F., Chang S.T. 2002. Comparisons of chemical charac- 
teristic variations for photodegraded softwood and hardwood with/without polyurethane clear coatings. Polym. Degrad. Stab. 77: 129-135.

Colom X., Carrillo F., Nogués F., Garriga P. 2003. Structural analysis of photodegraded wood by means of FTIR spectroscopy. Polym. Degrad. Stab. 80: 543-549.

Ferreira R.C., Lo Monaco A., Picchio R., Schirone A., Vessella F., Schirone B. 2012. Wood anatomy and technological properties of an endangered species: Picconia azorica (Oleaceae). IAWA J. 33 (4): 375-390.

Geladi P., Grahn H., Burger J. 2007. Multivariate images, hyperspectral imaging: background and equipment," Techniques and Applications of Hyperspectral Image Analysis, 1-15, Grahn H. and Geladi P. Eds., John Wiley \& Sons, West Sussex, England.

Genco G., Lo Monaco A., Pelosi C., Picchio R., Santamaria U. 2011. A study of colour change due to accelerated sunlight exposure in consolidated wood samples. Wood Res-Slovakia 56 (4): 511-524.

George B., Suttie E., Merlin A., Deglise X. 2005. Photodegradation and photostabilisation of wood - the state of the art. Polym. Degrad. Stab. 88: 268-274.

Kataoka Y., Kigushi M. 2001. Depth profiling of photo-induced degradation in wood by FT-IR Microspectroscopy. J. Wood Sci. 47: 325-327.

Hon D. N. S, Shiraishi N. 2001. Color and Discoloration and Weathering and photochemistry of wood. Wood and Cellulose chemistry, Marcel Dekker Inc., New York, 385-442, 513-546.

Lo Monaco A., Marabelli M., Pelosi C., Picchio R. 2011a. Colour measurements of surfaces to evaluate the restoration materials. Proc. SPIE 8084: 1-14

Lo Monaco A., Pelosi C., Picchio R. 2011b. Colour evolution of wood surfaces in simulated sunlight exposure. Proc. Colour and Colorimetry. Multidisciplinary Contributions, 207-214.

Marengo E., Robotti E., Liparota M. C., Gennaro M. C. 2003. A Method for Monitoring the Surface Conservation of Wooden Objects by Raman Spectroscopy and Multivariate Control Charts. Anal. Chem. 75: 5567-5574.

Marengo E., Robotti E., Liparota M. C., Gennaro. M. C. 2004. Monitoring of pigmented and wooden surfaces in accelerated ageing processes by FT-Raman spectroscopy and multivariate control charts. Talanta 63: $987-1002$

Moore A.K., Owen N.L. 2001. Infrared spectroscopic studies of solid wood. Appl. Spectrosc. 36: 65-86.

Nystrom J., Hagman 0. 1999. Methods for detecting compression wood in green and dry conditions. J. Wood Sci. 45(1): 30-37.

Noordam J.C, Van der Broek W.H.A.M., Geladi P., Buydens L.M.C. 2005. Detection and classification of latent defects and diseases on raw French fries with multispectral imaging. Chemom. Intell. Lab. Syst. 75(2): 115-126.

Otto M. 1999. Chemometrics, statistics and computer application in analytical chemistry. Wiley-VCH, New York.

Pandey K. K., Pitman A. J. 2003. FTIR studies of the changes in wood chemistry following decay by brown-rot and white-rot fungi. Int Biodeter. Biodegr. 52: 151-160.

Pandey K. K. 2005. Study of the effect of photo-irradiation on the surface chemistry of wood. Polym. Degrad. Stab. 90: 9-20.

Pelosi C., Agresti G., Calienno L., Lo Monaco A., Picchio R., Santamaria U., Vinciguerra V. 2013. Application of spectroscopic techniques for the study of the surface changes in poplar wood and possible implications in conservation of wooden artefacts. In: Proc. SPIE 8790-41: $1-14$.

Pillow M. 1941. A new method for detecting compression wood. $J$. Forest. 39: 385-387.

Thumm A., Riddell M., Nanayakkara B., Harrington J., Meder R. 2010. Near infrared hyperspectral imaging applied to ammping cemical composition in wood samples. J. Near Infrared Spectrosc. 18: 507515 .

Tolvaj L., Mitsui K., Varga D. 2011. Validity limits of Kubelka-Munk theory for DRIFT spectra of photodegradated solid wood. Wood Sci. Technol. 45: 135-146.

Van der Meer F.D., Jong S.M. (Eds.) 2002. Imaging Spectrometry: Basic Principles and Prospective Applications. Remote Sensing and Digital Image Processing, Vol. 4 Springer, Netherlands, 130-133. 